\title{
APPROACH TO
}

\author{
McGill Journal of Medicine
}

\section{Pediatric urinary tract infection (UTI)}

\author{
Maria Giannoumis $^{1}$
}

${ }^{1}$ Faculty of Medicine, McGill University, Montréal, Québec, Canada

\section{Correspondence}

Maria Giannoumis

Email: maria.giannoumis@mail.mcgill.ca

Publication Date

April 9, 2021

MJM 2021 (19) 18

https://doi.org/10.26443/mjm.v19i1.312

\section{क. McGill Journal of Medicine} www.mjmmed.com

\section{c) (1) (2)}

This work is licensed under a Creative Commons BY-NC-SA 4.0 International License.

\begin{abstract}
Urinary tract infections (UTIs) are prevalent in the children. Presentation of UTI vary in children of different ages. In infants, who cannot localize symptoms, UTI can present with a fever whereas in older children a UTI can present with urinary symptoms (dysuria, urinary frequency, incontinence). It is important to establish a clear diagnosis in order to treat and resolve the infection with antibiotics therapy to prevent bacteremia, pyelonephritis, and long-tern renal disease. Urine is collected through a mid-stream urine sample, in toilet trained children, via urethral catheterization, suprapubic aspiration and pediatric urine collection bags. Urine analysis and culture are the first-line investigations in children with suspected UTI. Goals of treatment include elimination of infection, relief of acute symptoms, and prevention of recurrent and long-term complications. The Canadian Pediatric Society recommends initial treatment with oral antibiotics for nontoxic children with febrile UTIs. Imaging, such as a renal/bladder ultrasound, may be used.
\end{abstract}

- KEYWORDS

Pediatric, Urinary Tract Infections, Clinical Approach

\section{1 | QUESTION}

Angela is a 10-month old girl presenting to your community emergency department. Past medical history includes a febrile urinary tract infection (UTI) at 6 months of age. She presents with a 2-day history of fever without diarrhea, vomiting or other symptoms. Her 6-yearold sister attends daycare and had a self-limiting febrile illness 1 week ago.
Angela is fussy but alert and consolable. On examination, she does not appear toxic. Her temperature is $39.1^{\circ} \mathrm{C}$. She is tachycardic with a heart rate of 155 beats/min with no audible murmur. Her respiratory rate is 34 breaths/min and she does not demonstrate laboured breathing or retractions, nor is she in distress. Bilaterally, her lungs are clear. She has no cough or congestion. Her abdomen is soft. Her tympanic membranes are normal and present with no nuchal rigidity. She does 
not have any rashes. Her genital examination findings are normal, with no erythema or labial adhesions. Identify the next best step in Angela's care:

A. Obtain a urine sample via catheterization for urinalysis and urine culture

B. Obtain a urine sample via bag collection for urinalysis and urine culture

C. No further investigations are required at this time, however a follow-up in 1 week is recommended if fever persists

D. Perform a voiding cystourethrogram (VCUG)

\section{ANSWER}

A. Angela requires a urine sample and further investigation. The appropriate next step is to obtain a urine sample through catheterization and send this urine sample for urinalysis and urine culture. Alternatively, one can use the bag method to obtain a sample for urinalysis, however it is not recommended to use bag-obtained samples for culture due to high contamination rates. Angela is 10 months old and presents with risk factors for UTI. Her risk factors include febrile illness without an identifiable source, young age, a history of UTI, and a temperature of greater than $39.0^{\circ} \mathrm{C}$ for 2 days. This presentation requires further investigation as a UTI is high on the differential diagnosis. A VCUG can be discussed with the family if vesicoureteral reflux (VUR) is suspected, however a renal bladder ultrasound (RBUS) is the recommended first-line investigation.

\section{3 | INITIAL APPROACH}

\section{1 | Pathogenesis and Classification}

UTIs frequently occur when bacteria invade and ascend up the urinary tract. Hematogenous spread to the urinary system is a rare cause of UTI that primarily occurs in neonates and immunodeficient children. (1) Lower UTIs are limited to cystitis, where the infection results in an inflammatory response in the bladder. Upper UTIs occur when the infection ascends to the ureters and kidneys resulting in pyelonephritis. (2) In previously healthy children who have not recently taken antibiotics, Escherichia coli account for the majority of UTIs. (3) Other common causative pathogens are Klebsiella, Proteus, and Enterococcus. (2-4) This approach to article discusses the diagnosis and management of uncomplicated UTIs in infants and children; uncomplicated UTIs are infections that are not an associated condition or structural abnormality.

UTIs are common bacterial infections in childhood that frequently cause acute illness in the pediatric population. (3) Occurrence rates among infants and children vary depending on age, sex, race, and circumcision status in males. (5) It is helpful to divide children into age groups to conceptualize approaches as clinical presentations, urine collection methods, and guidelines vary among children of different ages.

\section{2 | Infants and Toddlers 2 Months to 3 Years of Age}

\subsection{1 | Clinical presentation}

Approximately $7 \%$ of infants aged 2 to 24 months that present with fever without a source are diagnosed with a UTI. (5) Infants and toddlers frequently present late in the course of the infection with fever since they are unable to localize pain and urinary symptoms. When an infant or toddler presents with fever and no localizing symptoms (respiratory symptoms or rash suggestive of a viral etiology), the clinician must consider UTI as a cause. Recurrent UTIs should be further investigated as they can be indicative of complicated UTIs and associated with an underlying urinary tract abnormality, such as obstructive uropathies and VUR.

\subsection{2 | Recommendations}

The Canadian Pediatric Society (CPS) recommends that a urinalysis and urine culture be obtained from children less than 3 years of age who present with a fever $\left(>39.0^{\circ} \mathrm{C}\right.$ rectal) with no apparent source. (4) Children under 3 years of age who present with a fever greater than $39.0^{\circ} \mathrm{C}$ for more than 48 hours and without a lo- 
calizing source of fever are highly likely to have a UTI. Risk factors for UTIs can also be considered to calculate a pretest probability of UTI and determine which children require further investigation (Figure 1). It is important to consider other factors, such as the ability of the physician to follow up with the patient and the patient's history of UTIs. Additionally, the family unit should be included in the decision-making process.

\subsection{Children 3 Years of Age and Older}

\subsection{1 | Clinical presentation}

Older children are able to localize pain and describe symptoms. Symptoms such as dysuria, urinary frequency, new daytime incontinence, and suprapubic discomfort are common in cystitis and warrant testing for UTI. $(3,6)$ Prepubertal girls can present with dysuria and vulvovaginitis due to poor hygiene or exposure to irritants. History and physical exam are necessary to rule out non-UTI causes of dysuria. $(3,4)$ Urologic symptoms accompanied by systemic symptoms, flank pain, costovertebral tenderness, abdominal pain, and fever are suggestive of upper UTIs such as pyelonephritis. (7)

\subsection{2 | Recommendations}

The CPS states that the presence of urinary symptoms in verbal children, children usually greater than 3 years of age, can be used as a criterion for requesting further analysis and culture. (8) Children in this age group can generally submit to spontaneous urine samples for analysis and culture. (3)

\section{4 | Diagnosis}

In children presenting with a possible UTI, it is important to establish a clear diagnosis in order to treat and resolve the infection with appropriate antibiotic therapy. This prevents the spread of infection to the kidneys, longterm renal scarring and disease. UTI is diagnosed based on the results obtained from urine analysis and cultures. (3)

\subsection{1 | Urine collection}

In children who are toilet-trained, a mid-stream urine sample should be collected. In young children and those who cannot submit spontaneous samples, other means to obtain urine samples are required. Urethral catheterization, suprapubic aspiration (SPA) and pediatric urine collection bags are typically used. (9) It is important to consider that urine collection bags, although convenient and non-invasive, are at a high risk of contamination and are therefore not recommended for culture. (10) SPA is the gold standard for UTI diagnosis; any growth from a sterile SPA is diagnostic for a UTI. Table 1 summarizes advantages and disadvantages of available urine collection methods.

\subsection{2 | Urine Analysis}

Urine analysis is the physical, chemical (dipstick), and microscopic examination of urine. Urine dipsticks can be used to detect the presence of leukocyte esterase, a marker for white blood cells (WBCs) in the urine, and nitrites, a marker of gram-negative bacteria. The urine
Advantages

\begin{tabular}{ll} 
Urethral catheterization & $\begin{array}{l}\text { Sterile urine sample } \\
\text { Less discomfort than SPA }\end{array}$ \\
Suprapubic aspiration & $\begin{array}{l}\text { Gold standard for diagnosis of UTI } \\
\text { Sterile procedure }\end{array}$ \\
Bag Specimen & $\begin{array}{l}\text { Non invasive } \\
\text { Does not rely on technical skills }\end{array}$ \\
Clean catch void sample & $\begin{array}{l}\text { Non invasive } \\
\text { Does not rely on technical skills }\end{array}$ \\
\hline
\end{tabular}

Disadvantages

Invasive and uncomfortable

Less commonly used Invasive and painful

Not sterile, potential for contamination Not recommended for culture

Not sterile

Difficult to obtain in children who are not toilet-trained

TAB LE 1 Advantages and Disadvantages of Urine Collection Methods (3, 4, 9) 


\begin{tabular}{lll}
\hline Parameter & Sensitivity \%, (range) & Specificity \%, (range) \\
\hline & $83(67-94)$ & $78(64-92)$ \\
Leukocyte esterase test & $53(15-82)$ & $98(90-100)$ \\
Nitrite test & $93(90-100)$ & $72(58-91)$ \\
Leukocyte esterase or nitrite positive result & $73(32-100)$ & $81(45-98)$ \\
Microscopy, white blood cells & $81(16-99)$ & $83(11-100)$ \\
Microscopy, bacteria & $99.8(99-100)$ & $70(60-92)$ \\
\hline
\end{tabular}

TAB LE 2 Sensitivity and Specificity of Urine Analysis Components $(3,11)$

sample can be analyzed under a microscope to quantify the WBC concentration; 5 WBCs per high-power field is considered pyuria. (11) Infants empty their bladder more frequently, therefore gram-negative bacteria do not have the 4 hours required to form nitrites. For this reason, the nitrite test has a lower sensitivity in infants. The absence of nitrites does not rule out a UTI, however the test has a high specificity, thus the presence of nitrites is suggestive of UTI. Collecting information on the presence of WBCs and nitrites, as well as the WBC concentration, improves diagnostic testing for UTIs. Table 2 summarizes the sensitivities and specificities of urine analysis components.

\subsection{3 | Urine Culture}

Urine collection for culture must occur prior to starting antibiotics as a single dose of effective antibiotics can sterilize the urine. $(4,8)$ Bag collection methods are not reliable for culturing. For a clean catch specimen, $10^{5}$ colony forming units (CFU)/mL is indicative of a UTI. In a catheter specimen, $5 \times 10^{4} \mathrm{CFU} / \mathrm{mL}$ is indicative of a UTI. In these specimens, mixed growth is usually indicative of contamination and a repeat sample is recommended. Finally, in SPA, any growth is suggestive of a UTI as this is a sterile procedure. (4) It is important to consider the clinical presentation and the child's age when interpreting a culture result. $10^{4}-5 \times 10^{4}$ $\mathrm{CFU} / \mathrm{mL}$ may represent UTI in neonates, children with immunodeficiency, or children who have recently taken antimicrobial therapies. (3)

\section{5 | Management}

Goals of treatment include the elimination of the infection, relief of acute symptoms, and prevention of recurrent and long-term complications including hypertension, renal scarring, as well as impaired renal growth and function. (3) The CPS recommends initial treatment with oral antibiotics for nontoxic children with uncomplicated febrile UTIs (no structural abnormality) who can tolerate each dose. (4) Furthermore, the child should be treated empirically for the most likely bacterial pathogens while awaiting susceptibility test results, regardless of age. $(3,4)$ Therapy must be narrowed to the least broad-spectrum antibiotic when susceptibility results become available. Cefixime is a common antibiotic choice for outpatients presenting with their first febrile UTI. (4) Other common oral antibiotics are amoxicillin, amoxicillin/clavulanate, co-trimoxazole, cefprozil, and cephalexin. Other options include ampicillin, ceftriaxone, cefotaxime, gentamicin, and tobramycin. (11) Note that for complicated UTIs associated with a structural abnormality, IV antibiotic therapy is recommended. (4)

\section{4 | BEYOND THE INITIAL AP- PROACH}

\section{1 | Infants Less than 2 Months of Age and III-appearing children}

In the neonatal period, symptoms and signs are nonspecific. It is important to consider this age group carefully as children presenting with respiratory symptoms may 
have an appreciable risk of UTI. (3) Furthermore, in illappearing children with no other symptoms, the clinician should consider bacteremia and sepsis, as well as their appropriate work-ups. (4)

\section{2 | Role of Imaging and Other Investigations}

Imaging is not routinely used in children with cystitis. The objectives of imaging in the context of children with a UTI is to confirm pyelonephritis and identify VUR or other structural abnormalities of the urinary tract. (4) Currently, RBUS is commonly used in children and it is recommended as a standard tool in children $<2$ years of age with a first febrile UTI as it is non-invasive and inexpensive. (12) Previously, a VCUG was recommended in this age group. A VCUG is optimal for diagnosing and staging suspected VUR. A dimercaptosuccinic acid scan is the best tool for identifying an upper UTI and renal scarring, however it exposes patients to radiation and is therefore not routinely performed unless results may alter treatment. (13) Laboratory tests such as procalcitonin and C-reactive protein can be measured; if elevated, they can suggest renal involvement.

\section{REFERENCES}

1. Schlager TA. Urinary tract infections in children younger than 5 years of age: epidemiology, diagnosis, treatment, outcomes and prevention. Paediatr Drugs. 2001;3(3):219-27.

2. Simões e Silva AC, Oliveira EA. Update on the approach of urinary tract infection in childhood. J Pediatr (Rio J). 2015;91 (6 Suppl 1):S2-10.

3. Balighian E, Burke M. Urinary Tract Infections in Children. Pediatr Rev. 2018;39(1):3-12.

4. Robinson JL, Finlay JC, Lang ME, Bortolussi R. Urinary tract infections in infants and children: Diagnosis and management. Paediatr Child Health. 2014;19(6):315-25.

5. Shaikh N, Morone NE, Bost JE, Farrell MH. Prevalence of urinary tract infection in childhood: a meta-analysis. Pediatr Infect Dis J. 2008;27(4):302-8.

6. Leung AKC, Wong AHC, Leung AAM, Hon KL. Urinary Tract Infection in Children. Recent Pat Inflamm Allergy Drug Discov. 2019;13(1):2-18.

7. Morello W, La Scola C, Alberici I, Montini G. Acute pyelonephritis in children. Pediatr Nephrol. 2016;31(8):1253-65.
8. Shaikh N, Morone NE, Lopez J, Chianese J, Sangvai S, D'Amico $\mathrm{F}$, et al. Does this child have a urinary tract infection? Jama. 2007;298(24):2895-904.

9. May OW. Urine Collection Methods in Children: Which is the Best? Nurs Clin North Am. 2018;53(2):137-43.

10. Al-Orif F, McGillivray D, Tange S, Kramer MS. Urine culture from bag specimens in young children: are the risks too high? J Pediatr. 2000;137(2):221-6.

11. Roberts KB. Urinary tract infection: clinical practice guideline for the diagnosis and management of the initial UTI in febrile infants and children 2 to 24 months. Pediatrics. 2011;128(3):595-610.

12. Hoberman A, Charron M, Hickey RW, Baskin M, Kearney DH, Wald ER. Imaging studies after a first febrile urinary tract infection in young children. N Engl J Med. 2003;348(3):195-202.

13. Zhang X, Xu H, Zhou L, Cao Q, Shen Q, Sun L, et al. Accuracy of early DMSA scan for VUR in young children with febrile UTI. Pediatrics. 2014;133(1):e30-8.

14. Leroy S, Fernandez-Lopez A, Nikfar R, Romanello C, Bouissou F, Gervaix A, et al. Association of procalcitonin with acute pyelonephritis and renal scars in pediatric UTI. Pediatrics. 2013;131(5):870-9. 


\section{5 | TABLES \& FIGURES}

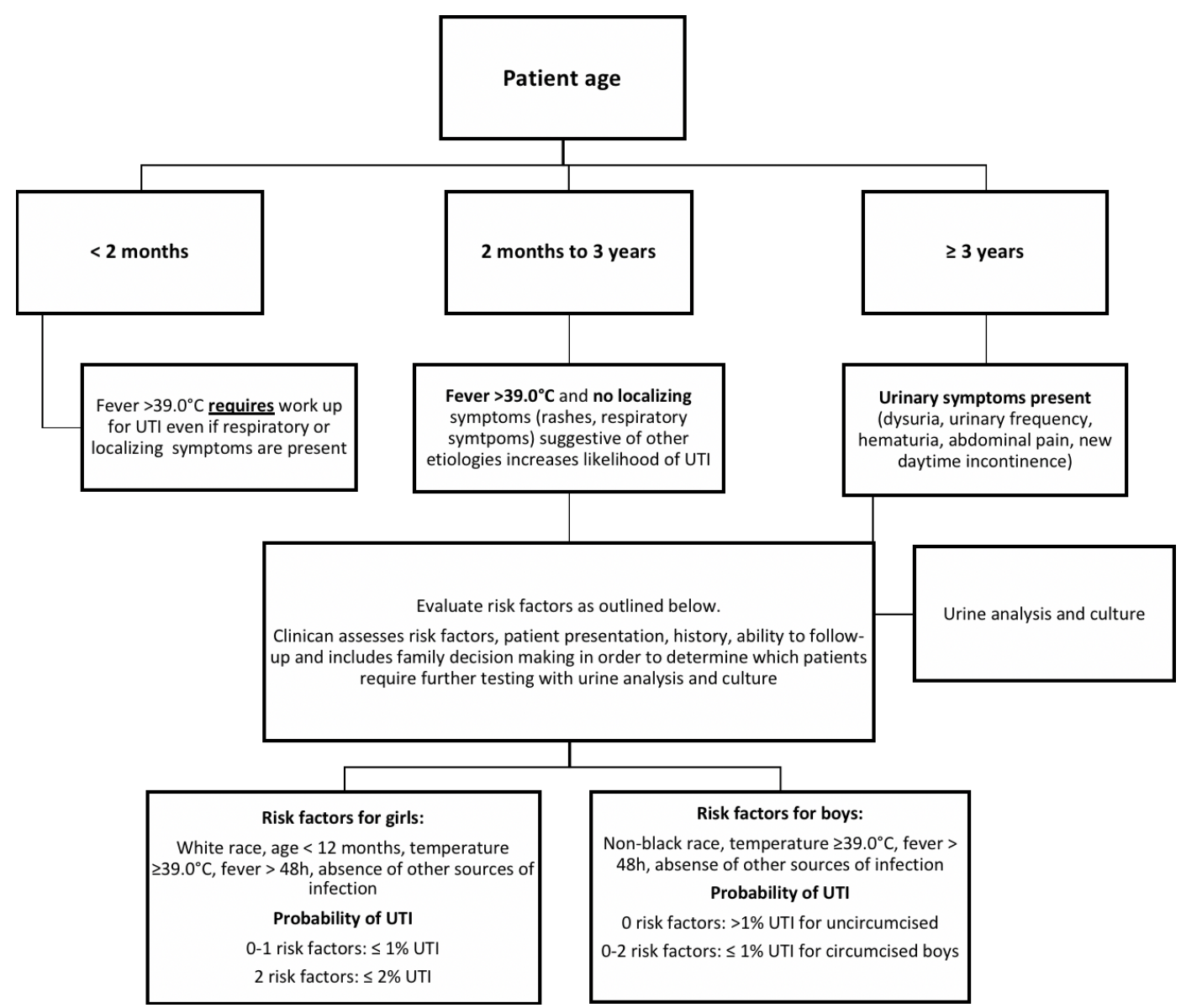

FLOWCHART 1 Approach to UTls in infants and children of different ages 